\title{
Ethical dilemmas in paediatric intensive care in the South African public healthcare sector
}

\author{
D E Ballot, ${ }^{1}$ PhD, MB BCh, FCPaeds; T D Ramdin, ${ }^{1}$ MB BCh, DCH, FCPaeds, MMed, Cert Neonatology; \\ D A White, ${ }^{1}$ MB BCh, FCPaeds, MMED, Cert Pulmonology, Dip Allergology; A Dhai, ${ }^{2}$ PhD, MB ChB, FCOG, LLM, PG Dip (Int Res Ethics)

\begin{abstract}
${ }^{1}$ Paediatric/Neonatal Intensive Care Unit, Charlotte Maxeke Johannesburg Academic Hospital and Department of Paediatrics, Faculty of Health Sciences, University of the Witwatersrand, Johannesburg, South Africa

${ }^{2}$ Steve Biko Centre for Bioethics, Faculty of Health Sciences, University of the Witwatersrand, Johannesburg, South Africa
\end{abstract}

Corresponding author: D Ballot (Daynia.ballot@wits.ac.za)

\begin{abstract}
The shortage of resources in the public healthcare sector in South Africa (SA) means that healthcare providers face difficult choices in terms of rationing of care. This is particularly challenging in paediatrics. This article reviews the ethical and legal issues that arise in this regard. Principlism is the ethical framework most commonly used by clinicians, but perhaps other approaches, such as the ethics of caring, and ubuntu, would be more appropriate for the SA situation.
\end{abstract}

SAfr J Bioethics Law 2019;12(1):44-46. DOI: 10.7196/SAJBL.2019.v12i1.672

Ethical problems are frequently confronted in the realm of paediatric intensive care. High-income countries (HICs) have more critical care beds, paediatric intensivists and paediatric intensive care unit (PICU) admissions than low- and middle-income countries (LMICs), ${ }^{[1]}$ where the majority of deaths in children under 5 years of age occur. ${ }^{[1]}$ In addition to these advantages, healthcare expenditure in HICS is USD1 060 per capita annually, compared with USD98 in sub-Saharan Africa. ${ }^{[2]}$ The provision of PICUs in LMICs is hampered by difficulties in obtaining finances, a lack of appropriately trained nurses and doctors, a lack of equipment and a shortage of blood products and sedatives. ${ }^{[1]}$ South Africa (SA) is a middle-income country with limited resources and a shortage of ICU beds. A descriptive study in 2007 showed that only $23 \%$ of public sector hospitals had ICU or high care beds, compared with $84 \%$ of hospitals in the private sector. This translates to $1783 \mathrm{ICU} /$ high care beds in the public sector, which serves $\sim 80 \%$ of the population, v. 2385 beds in the sector serving under $20 \% \cdot{ }^{[3]}$ At the time of the study, only $19.6 \%$ of the available ICU beds were dedicated to paediatric or neonatal patients. ${ }^{[3]}$ In SA, the disparity between public and private sector resources adds to the intensivist's dilemma, because rationing, withholding and withdrawal of care are routine for the majority of the population, but do not generally apply to those who can afford private healthcare. The implementation of National Health Insurance (NHI) aims to reduce such disparities.

In resource-constrained settings, the limited availability of PICU beds is often managed through rationing care, where patients with the best anticipated outcome are admitted to ICU, often at the expense of other patients. ${ }^{[4]}$ Another possible approach would be to treat patients with a better anticipated outcome in level 2 wards, outside of the ICU, so that resources can be concentrated on those with a poorer prognosis. As the circumstances and available resources in healthcare in HICs are so different from those in SA, HIC guidelines are difficult to apply in the local context.
In this article we discuss some of the key ethical challenges frequently faced by PICU practitioners in LMICs. We also highlight the ethicolegal/clinical-reality paradox in SA. While section 28(2) of the SA Constitution ${ }^{[5]}$ requires that a child's best interests are considered paramount whenever any decision is made for that child, the state of affairs is such that clinicians have to make decisions based on the realities on the ground.

\section{Ethical challenges: Withholding or withdrawal of care}

Withholding or withdrawal of care is frequently practiced in the PICU. The Royal College of Paediatrics and Child Health (RCPCH) in the UK states that there are five circumstances in which this may be appropriate: $:^{[6]}$

- brain death

- persistent vegetative state - where the child is reliant on others for all functions and does not interact with the environment

- no-chance situation - where the process of dying is prolonged without relief of suffering

- no-purpose situation - where the child would survive treatment with extremely severe physical or mental handicap

- unbearable situation - where the life-saving treatment is too much to be borne.

Some authors refer, in this context, to both futile and inappropriate care. ${ }^{[7]}$ Care is considered futile when there is no chance of achieving any physiological goal. Inappropriate care is that in which there is only a small chance of achieving the planned outcome, or the outcome is considered to be unreasonable, e.g. prolonged ventilation of a person declared brainstem dead. Generally, it is recommended that neither futile nor inappropriate care should be initiated or continued, irrespective of the availability of resources. This would be in line with respecting the inherent dignity of the child, and the ethical principle 
of non-maleficence. Decisions regarding withholding or withdrawal of futile or inappropriate care need to be made on an individual basis and to include input from the clinical team and the patient's family. In the main, the bioethical principles of autonomy and beneficence are used for ethical analysis in this process. ${ }^{[7]}$ In cases that are not clear, admission to and stabilisation in PICU with review of the patient after 24 - 48 hours is preferable. At this point, it may be apparent that medical intervention is now futile or inappropriate, and decisions can be guided accordingly.

Although there is no legal or ethical distinction between withholding and withdrawal of care, it is psychologically more difficult to withdraw care.$^{[8]}$ It is frequently difficult for parents to participate in a decision to stop treatment of their child, and an approach of 'non-dissent' may be preferable, where the parents are informed of the reasonably available treatment options and are given a chance to dissent with the treatment plan. ${ }^{[8]}$ This could pose a challenge where parents and patients have trouble understanding the treatment options, because of language barriers or cultural or traditional beliefs. Where the recommended treatment is in the best interests of the child, even if this means withdrawal of care, and the parents and family disagree, review by an institutional board is recommended. ${ }^{[8]}$ In the SA context, this would equate to hospital ethics committees. In reality, hospital ethics committees are unlikely to be readily available in public sector hospitals in SA, owing to a paucity of clinical ethicists, and the courts should be a last resort.

Respect for autonomy means that those capable of deliberation around personal choices be treated with respect for their capacity of self-determination. Persons with diminished or impaired autonomy, i.e. those who are dependent or vulnerable, must be protected and afforded security against harm or abuse. ${ }^{[9]}$ Children are considered both dependent and vulnerable because of their unique physiological and psychosocial status. In addition, they lack legal capacity to consent. While the Children's Act No. 38 of $2005^{[10]}$ allows for children aged 12 years and above to consent to medical procedures on condition that they are mature enough to do so, and in the case of surgical procedures, as an additional safeguard, aided by parents or guardians, the reality is that most children in the PICU, irrespective of age, are not in a frame of mind to make informed decisions. The cutoff age for PICU is generally around $12-14$ years in the public sector hospitals in Gauteng Province where the authors practice. Parents, or legal guardians, are the usual decision-makers for minor children (under 18 years of age), and are required to consider the child's best interests (grounded in the principle of beneficence, and affirmed in the Constitution). However, to find an acceptable option, parents may be required to consider competing interests of other family members. Where possible, assent must be obtained from children who are old enough to participate in the process ${ }^{[8]}$ The guidelines of the $\mathrm{RCPCH}$ in the UK state that an older child with sufficient maturity and extensive experience of illness may competently consent to the withdrawal or withholding of lifesaving treatment. ${ }^{[6]}$ It is also important to bear in mind that $\mathrm{SA}$ is a diverse and divided nation of multiple racial and ethnic groups, languages and cultures, with huge disparities in socioeconomic status and literacy. All these factors have an impact on the perspectives of both healthcare providers and patients, which may, for example, result in differing understandings of the disease process. $^{[11]}$

\section{Rationing challenges}

$\mathrm{SA}$, as a middle-income country, has limited health resources and a critical shortage of PICU beds. Paediatric ICU is frequently provided in shared units - either in adult ICUs or together with neonatal ICUs. Rationing of care has been practised for a long time, but the criteria have changed over the decades. Argent et al. ${ }^{[12]}$ have developed admission guidelines for PICUs in SA. The exclusion criteria (for withholding ICU care) are similar to the RCPH criteria, and include futile care, underlying lethal conditions and 'conditions with a currently poor outcome. This last includes patients who have deteriorated on appropriate treatment after more than 5 days of hospital admission. ${ }^{[12]}$

Charlotte Maxeke Johannesburg Academic Hospital has a combined paediatric and neonatal ICU, and the admission criteria for neonates affect the overall availability of ICU beds. Based on justice considerations, birth weight has long been used to ration admission to NICU in SA. Unlike the RCPCH guidelines, this approach denies care to some children who are potentially viable. In the 1990s when NICU was first established in SA, neonates weighing $<1000 \mathrm{~g}$ were not provided with any ventilatory support. The basis for this approach was the concern that ventilating a very small/preterm neonate would occupy an ICU bed for a prolonged period, potentially saving an extreme preterm neonate at the expense of two or three equally viable neonates of larger birthweight. Advances in neonatal care, particularly the advent of non- invasive ventilation (nasal continuous positive airway pressure (NCPAP)) and surfactant therapy, which are provided in high care wards, as opposed to NICU, have allowed more liberal weight cut-offs to be implemented. Currently, in this study unit, neonates $>750 \mathrm{~g}$ at birth are provided with surfactant replacement therapy and NCPAP, while those $>900 \mathrm{~g}$ are provided with mechanical ventilation. ${ }^{[13]}$ Provision of NCPAP in high care wards, however, requires additional resources and staffing. It is also important to be aware of possible unintended consequences, such as the ethical dilemma to be faced when a $750 \mathrm{~g}$ neonatal survivor requires ventilation for pneumonia. In addition, extreme preterm neonates are at risk of major complications such as intraventricular haemorrhage, with potential neurodevelopmental handicap. It is therefore important to monitor long-term outcomes when considering ventilation policies in neonatal care.

There have been similar changes over time in PICU admission policies in SA. Prior to the implementation of highly active antiretroviral therapy, HIV-infected children had an extremely high mortality and were not admitted to PICU. In a recent review of medical admissions to PICU in Johannesburg, however, one-third of all children were HIV-exposed, and had similar outcomes to their HIVnegative counterparts. ${ }^{[14]}$ Concurrent infection with cytomegalovirus in HIV-exposed children with lower respiratory tract infections and respiratory failure was found to be associated with worse outcomes, so routine ganciclovir therapy is now recommended in these patients. ${ }^{[15]}$ Infection with HIV is therefore no longer an exclusion criterion for PICU admission in SA.

Inequalities, together with increasing healthcare costs, inevitably stimulate debate on the role of justice and its requirements in particular societies, with notions of fairness and entitlement being central to these discussions. ${ }^{[16]}$ In the context of rationing, distributive justice, which refers to 'fair, equitable and appropriate distribution of 
benefits and burdens, determined by norms that structure the terms of social co-operation,' applies. ${ }^{[17]}$

Decisions about PICU management are made at both macro and micro levels. Justice, priority-setting and rationing are macrolevel determinations, and, out of necessity, must consider resource constraints. These macro-level decisions are usually informed by utilitarian principles. At the level of the practitioner-patient relationship (the micro level), while principlism seems to be the most widely used concept, dilemmas in clinical decision-making often arise because of the disconnect between the macro-level utilitarian approach and the core values of caring and compassion in clinical practice. In addition, other values, such as truthfulness, integrity and respect for religious and cultural differences, also come into play. In the SA cross-cultural context, the ethics of caring, which encompass sensitivity, honesty, patience, trustworthiness, respect, compassion and trust in all interactions, may be a better approach than principlism. ${ }^{[11]}$ In addition, this is more in keeping with the concept of ubuntu, the humane treatment of others. ${ }^{[18]}$

\section{The ethicolegal/clinical-reality paradox}

Section 28(1)(c) of the SA Constitution ${ }^{[5]}$ affirms the right of all children to basic healthcare services. However, admission into PICU is dependent on the availability of beds, and hence the reality is that many children are denied this Constitutional protection. Section 28(2) establishes that a child's interests are of paramount importance in every matter concerning the child. The Children's Act ${ }^{[10]}$ was promulgated by the state to protect and promote this right. Section 6(2) of the Act lays down a number of principles to be observed when dealing with children. These include respect for the child's inherent dignity, fair and equitable treatment, and recognising the child's need for development. Severely limited resources, together with the need to balance the competing interests of other family members, are frequently inconsistent with the rights and principles espoused by the Constitution and the Act. Furthermore, the Act provides 14 factors that need to be considered when applying the 'best interests of the child'standard. However, while these factors might be of value in the child's social sphere, they are of marginal benefit to the PICU practitioner in the clinical context, where decisions have to be made based on the realities and lived experiences of all those affected on the ground, i.e., both the patient and the treatment team.

\section{Conclusion}

Ethical challenges are both common and complex in PICUs. SA, like other LMICs, has no option but to function within the limitations imposed by constrained resources. Critical care guidelines should not be translated from HICs to LMICs; instead, there is a need for good-quality research from LMICs to inform health policies and develop guidelines appropriate for local resources and spectrums of disease. ${ }^{[19]}$ The ethical framework for these guidelines should also be appropriate for the LMICs context. Moreover, while principlism is readily understood by medical practitioners because it lends itself easily to an empirical approach, other ethical frameworks such as the ethics of caring, and ubuntu, may be preferable in the diverse contexts of SA. Most importantly, however, the paradox that arises out of the contrast between ethicolegal mandates and harsh realities at the level of provision of care must be recognised and confronted as a critical contributory factor to the ethical dilemmas faced by practitioners in PICUs in SA. The implementation of $\mathrm{NHI}$ may, with time, address some of the resource constraints and disparities in PICUs between the public and private sectors, but this is not likely to be a quick or an easy process.

Acknowledgements. None.

Author contributions. Equal contributions.

Funding. None.

Conflicts of interest. None.

1. Tripathi S, Kaur H, Kashyap R, Dong Y, Gajic O, Murthy S. A survey on the resources and practices in paediatric critical care of resource-rich and resource-limited countries. J Intensive Care 2015;3:40. https://doi.org/10.1186/s40560-015-0106-3

2. Argent AC, Chisti MJ, Ranjit S. What's new in PICU in resource-limited settings? Intensive Care Med 2018;44(4):467-469. https://doi.org/10.1007/s00134-0174905-y

3. Bhagwanjee S, Scribante J. National audit of critical care resources in South Africa - unit and bed distribution. S Afr Med J 2007;97(12 Pt 3):1311-1314.

4. Manda-Taylor L, Mndolo S, Baker T. Critical care in Malawi:The ethics of beneficence and justice. Malawi Med J 2017;29(3):268-271. https://doi.org/10.4314/mmj. v29i3.8

5. South Africa. Constitution of the Republic of South Africa, 1996.

6. Larcher V, Craig F, Bhogal K, Wilkinson D, Brierley J. Making decisions to limit treatment in life-limiting and life-threatening conditions in children: A framework for practice. Arch Dis Child 2015;100(Suppl 2):s3-s23. https://doi.org/10.1136/ archdischild-2014-306666

7. Rubin MA, Truog RD. What to do when there aren't enough beds in the PICU. AMA J Ethics 2017;19(2):157-163. https://doi.org/10.1001/journalofethics.2017.1 9.2.ecas3-1702

8. Orioles A, Morrison WE. Medical ethics in paediatric critical care. Crit Care Clin 2013;29(2):359-375. https://doi.org/10.1016/j.ccc.2012.12.002

9. Council for International Organizations of Medical Sciences. International Ethical Guidelines for Biomedical Research Involving Human Subjects. Geneva: CIOMS, 2002. 10. South Africa. Children's Act No. 38 of 2005.

11. Betancourt JR, Green AR, Carrillo JE. The challenges of cross-cultural healthcare diversity, ethics and the medical encounter. Bioethics Forum 2000;16(3):27-32.

12. Argent AC, Ahrens J, Morrow BM, et al. Pediatric intensive care in South Africa: An account of making optimum use of limited resources at the Red Cross War Memorial Children's Hospital. Pediatr Crit Care Med 2014;15(1):7-14. https://doi. org/10.1097/PCC.0000000000000029

13. Ballot DE, Chirwa T, Ramdin T, et al. Comparison of morbidity and mortality of very low birth-weight infants in a central hospital in Johannesburg between 2006/2007 and 2013. BMC Pediatr 2015;15:20. https://doi.org/10.1186\%2Fs12887-015-0337-4

14. Mopeli R, Ballot DE, White DA. An audit of primary medical conditions in children admitted to the paediatric intensive care unit of Charlotte Maxeke Johannesburg Academic Hospital. S Afr J Child Health 2016;10(4):221-226. https://doi.org/10.7196/ SAJCH.2016.v10i4.1187

15. Kitchin OP, Masekela R, Becker P, Moodley T, Risenga SM, Green RJ. Outcome of human immunodeficiency virus-exposed and -infected children admitted to a paediatric intensive care unit for respiratory failure. Pediatr Crit Care Med 2012;13(5):516-519.

16. World Health Organization. Making fair choices on the path to universal health coverage. Geneva: WHO, 2014. https://www.who.int/choice/documents/ making fair choices/en/ (accessed 1 March 2019).

17. Beauchamp TL, Childress JF. Justice. In: BeauchampTL, Childress JF, eds. Principles of Biomedical Ethics. New York: Oxford University Press, 2013:249-301.

18. Bennet TW. Introduction. In: Bennet TW, Munro AP, Jacobs, PL, eds. Ubuntu: An African Jurisprudence. Cape Town: Juta (Pty) Ltd, 2018:1-7.

19. Von Saint Andre-von Arnim AO, Attebery J, Kortz TB, et al. Challenges and priorities for pediatric critical care clinician-researchers in low- and middle-income countries. Front Pediatr 2017;5:277. https://doi.org/10.3389/fped.2017.00277

Accepted 22 May 2019. 